\title{
The effects of the small- $t$ properties of hadronic scattering amplitude on the determination its real part
}

\author{
O. V. Selyugin* \\ BLTPh, Joint Institute for Nuclear Research, Dubna, Russia
}

\begin{abstract}
Taking into account the different forms of the Coulomb-hadron interference phase and the possible spin-flip contribution the new analysis of the experimental data of the proton-antiproton elastic scattering at $3.8<p_{L}<6.0 \mathrm{GeV} / \mathrm{c}$ and small momentum transfer is carried out. It is shown that the size of the spin-flip amplitude can be determined from the form of the differential cross sections at small $t$, and the deviation of $\rho(s, t)$ obtained from the examined experimental data of the $p \bar{p}$ scattering from the analysis [1], based on the dispersion relations, is conserved in all examined assumptions. The analysis of the proton-proton elastic scattering at $9<p_{L}<70 \mathrm{GeV} / \mathrm{c}$ also shows the impact of the examined effects on the form of the differential cross sections.
\end{abstract}

\section{Introduction}

Hadronic high-energy physics has progressed tremendously since the early days of the S-Matrix theory and of Regge poles. Initially, it was believed that the imaginary part of the (spin-non-flip) amplitude was what really mattered in the forward direction because of the constraints coming from unitarity [2]. Eventually, this led to the birth and growth of the pomeron philosophy. As time went by and the analysis got more and more refined and higher and higher energies were explored, people realized that the actual picture was considerably more complex. Analyticity soon showed that one could not do without a real part [3, 4] while polarization data proved that it was not possible to ignore spin complications. The long believed axiom that "spin complications disappear at asymptotic energies" has (so far) never found confirmation; alternatively, the asymptotic domain has never been reached (yet). In fact, it was shown that the spinflip amplitude gives a non negligible contribution and indeed it was the polarization data that led to the first decline of the simple minded Regge pole picture.

The measure of the $s$-dependence of the total cross sections $\sigma_{\text {tot }}(s)$ and of $\rho(s, t)$ - the ratio of the real to imaginary part of the elastic scattering amplitude is very important as they are connected to each other through the integral dispersion relations

$$
\rho_{\frac{p p}{p \bar{p}}}(E) \sigma_{\frac{p p}{p \bar{p}}}(E)=\frac{A \frac{p p}{p \bar{p}}}{p}+\frac{E}{\pi p} \int_{m}^{\infty} d E^{\prime} p^{\prime}\left[\frac{\sigma \frac{p p}{p \bar{p}}\left(E^{\prime}\right)}{E^{\prime}\left(E^{\prime}-E\right)}-\frac{\sigma_{\frac{p \bar{p}}{p p}}\left(E^{\prime}\right)}{E^{\prime}\left(E^{\prime}+E\right)}\right] .
$$

*selugin@theor.jinr.ru 

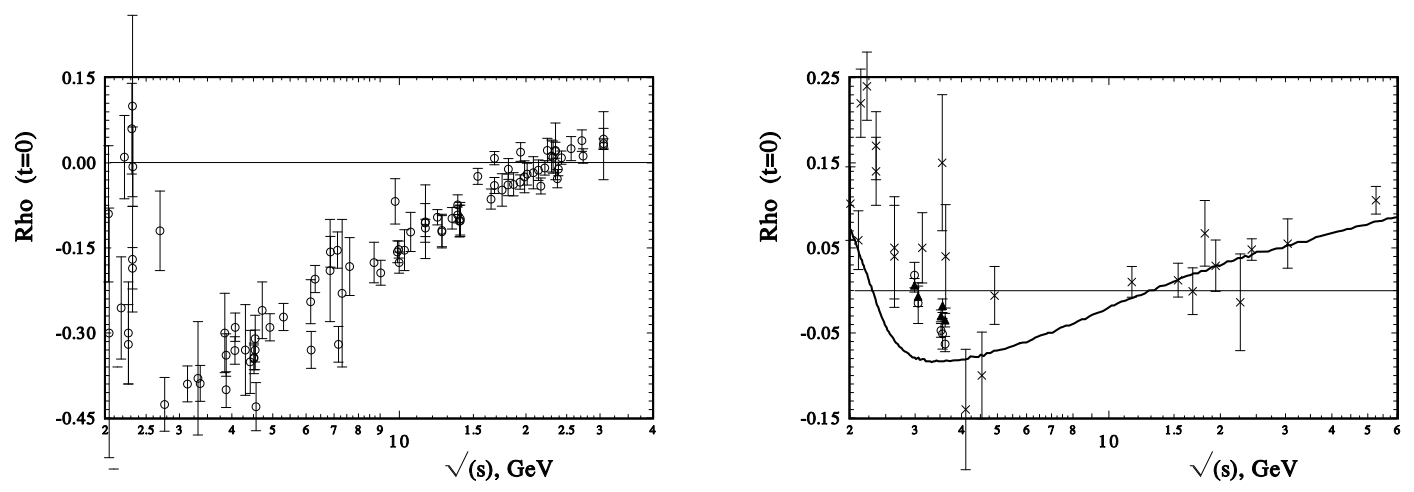

Figure 1: $\quad \rho(s, t=0)$ - the ratio of the real to imaginary part of the elastic scattering amplitude of a(top plot) for $p p$ scattering [experimental points - the world database); with curves of the different models and $\mathrm{b}$ (bottom plot) for $p \bar{p}$ scattering at low energies (triangles - the fit with fixed $\sigma_{t o t}$ and circles - the fit with "free $\sigma_{t o t}$ " the curve is the dispersion relation description for the $p \bar{p}$ scattering

The validity of this relation can be checked at LHC energies [5]. The deviation can point out to the existence of a fundamental length at $\mathrm{TeV}$ energies [6, 7]. But for such a conclusion we should know with high accuracy the lower energy data as well. The difficulty is that we do not know the energy dependence of these amplitudes and individual contributions of the asymptotic non-dying spin-flip amplitudes. As was noted in [8], the spin-dependent part of the interaction in $p p$ scattering is more important than expected and a good fit to the data in the Regge model requires an enormous number of poles.

As we do not know exactly, from a theoretical viewpoint, the dependence of the scattering amplitude on $s$ and $t$ one, it is usually assumed that the imaginary and real parts of the spin-nonflip amplitude behave exponentially with the same slope. Similarly, one assumes the imaginary and real parts of the spin-flip amplitudes (without the kinematic factor $\sqrt{|t|}$ ) to have an analogous $t$-dependence in the examined domain of momenta transfer. Moreover, one assumes energy independence of the ratio of spin-flip to spin-non-flip parts at small $t$. All this is our theoretical uncertainty.

In this paper we consider in the greatest detail the situation concerning $\rho(s, t)$. The model we propose takes into account all known features of the near forward proton proton and proton antiproton data, slopes of the spin-non-flip and of spin-flip amplitudes, total cross sections, ratios of the real to the imaginary forward amplitudes and Coulomb-nuclear interference phase where the form factors of the nucleons are also considered. Our main difficulty is due to the lack of experimental data at high energies and small momenta transfer with high accuracies.

The non-trivial procedure of the extraction of the size of $\rho(s, t)$ from the experimental data on the differential cross sections shows the semi-phenomenological properties of $\rho(s, t)$. Its size is dependent on some theoretical assumption 9]. For example, a significant discrepancy in the experimental measurement of $\rho$ was found by the UA4 and UA4/2 collaborations at $\sqrt{s}=541 \mathrm{GeV}$. But a more careful extrapolation [10] to $t=0$ shows that there is no real contradiction between these measurements and gives for this energy $\rho(\sqrt{s}=541 \mathrm{GeV}, t=0)=0.163$, the same as in the previous phenomenological analysis [11]. 
Of course, we have plenty of experimental data in the domain of small $t$ at low energies $3<p_{L}<100(\mathrm{GeV} / \mathrm{c})$. Unfortunately, most of these data come with large errors. The extracted sizes of $\rho(s, t=0)$ contradict each other in the different experiments (Fig.1a) and give a bad $\chi^{2}$ in the different models trying to describe the $s$-dependence of $\rho(s, t=0)$ (see, for example, the results of the COMPETE Collaboration [12, 13, 14]). It is of first importance that a more careful analysis of these experimental data gives in some cases an essentially different extrapolation for $\rho(s, t=0)$. For example, the analysis of the experimental data made in [11, which takes into account the uncertainty of the total cross sections (3-parameters fit) and the uncertainty of the Luminosity (4-parameters fit) gave a $\rho(s, t=0)$, which differs from the original values obtained by the experimental group, by $25 \%$ on average. For example, for $p_{L}=19.23 \mathrm{GeV} / \mathrm{c}$ the experimental work gave $\rho(s, t=0)=-0.25 \pm 0.03$ and for $p_{L}=38.01 \mathrm{GeV} / \mathrm{c}$ $\rho(s, t=0)=-0.17 \pm 0.03$. The analysis with free 4-parameters gave for these values: $\rho(s, t=0)=-0.32 \pm 0.08$ and $\rho(s, t=0)=-0.12 \pm 0.03$, respectively. This kind of picture was confirmed by the independent analysis of the experimental data [15, 16] $52<p_{L}<400(\mathrm{GeV} / \mathrm{c})$ of Fajardo [17] and Selyugin [11]. Both new analyses coincide with each other but differ from the original experimental determination.

The data on proton-antiproton elastic scattering at $3.7<p_{L}<6.2(\mathrm{GeV} / \mathrm{c})$ are most interesting. These experimental data have high accuracies and give the extracted value of $\rho(s)$ with high precision [18]. On Fig.1(b) these data are shown together with other experimental data and the predictions of the dispersion analysis carried out by Kroll [1]. The data for $\rho(s)$ essentially differ from the theoretical analysis. Hence, a more careful analysis of the original experimental data is required to take into account the different assumptions and corrections to the scattering amplitude.

In this paper we will examine how different effects can influence the determination of $\rho(s, t)$. First we will consider the effect of the Coulomb-hadron interference phase (sections 2 and 3). Then we will consider spin effects (sections 4 and 5) and then, briefly, the effect of oscillations (section 6).

\section{The Coulomb phase and the hadron form- factor}

The electromagnetic amplitude can be calculated in the framework of QED. In the one-photon approximation and the high energy approximation, it can be obtain [19] for the spin-non-flip amplitudes:

$$
F_{1}^{e m}=\alpha f_{1}^{2} \frac{s-2 m^{2}}{t}, \quad F_{3}^{e m}=F_{1}^{e m}
$$

and the spin-flip amplitudes:

$$
F_{4}^{e m}=-F_{2}^{e m}, \quad F_{5}^{e m}=\alpha \frac{s}{2 m \sqrt{|t|}} f_{1} f_{2} .
$$

where $f_{1}(t)$ and $f_{2}(t)$ are the Sachs form factors of the proton

$$
f_{1}(t)=\frac{4 m^{2}-t(1+k)}{4 m^{2}-t} G_{d}, \quad f_{2}(t)=\frac{4 m^{2} k}{4 m^{2}-t} G_{d}
$$

with $G_{d}=\left(1-t / \Lambda^{2}\right)^{-2}$; where $t=-q^{2} \mathrm{GeV}^{2}$ is the momentum transfer, $\Lambda^{2}=0.71$ $\mathrm{GeV}^{2}, m$ is the proton mass, and $k=1.793$ is the anomalous magnetic moment of the proton. 
The differential cross sections of nucleon-nucleon elastic scattering can be written as the sum of different helicity amplitudes:

$$
\frac{d \sigma}{d t}=\frac{2 \pi}{s^{2}}\left(\left|\phi_{1}\right|^{2}+\left|\phi_{2}\right|^{2}+\left|\phi_{3}\right|^{2}+\left|\phi_{4}\right|^{2}+4\left|\phi_{5}\right|^{2}\right) .
$$

Including electromagnetic and hadronic interactions, every amplitude $\phi_{i}(s, t)$, can be expressed as

$$
\phi_{i}(s, t)=F_{i}^{e m} \exp (i \alpha \varphi(s, t))+F_{i}^{h}(s, t)
$$

where

$$
\varphi(s, t)=\varphi_{C}(t)-\varphi_{C h}(s, t) .
$$

$\varphi_{C}(t)$ will be calculated in the second Born approximation in order to allow the evaluation of the Coulomb-hadron interference term $\varphi_{C h}(s, t)$. The quantity $\varphi(s, t)$ has been calculated and discussed by many authors. For high energies, the first results were obtained by Akhiezer, Pomeranchuk [20] for the diffraction on a black nucleus. Using the WKB approach in potential theory, Bethe [21] derived $\varphi(s, t)$ for proton-nucleus scattering

$$
\varphi(s, t)=2 \ln (1.06 / q a),
$$

where the parameter, $a$, characterizing the range of the strong-interaction forces is defined by the size of a nucleus. After some improvement [22, 23], the most important result was obtained by Locher [24] and then by West and Yennie using the Feynman diagram technique [25]. If the hadron amplitude has the usual Gaussian form

$$
F^{h}(s, t)=h_{n f}(s) \exp \left(-B(s) q^{2} / 2\right),
$$

where $h_{n f}$ denotes the "non-flip" amplitude, one gets the standard phase which is used in most experimental works,

$$
\varphi(s, t)=\mp[\ln (-B(s) t / 2)+\gamma]
$$

where $B(s) / 2$ is the slope of the nuclear amplitude, $\gamma$ is the Euler constant, and the upper (lower) sign corresponds to the scattering of particles with the same (opposite) charges.

The influence of the electromagnetic form factor of the scattered particles on $\varphi_{C h}$ in the framework of the eikonal approach was examined by Cahn [26]. He derived for $t \rightarrow 0$ the eikonal analogue of [25] and obtained the phase, with take into account the form factor in the monopole form,

$$
\begin{aligned}
\varphi_{C h}(s, t)= & \mp\left[\gamma+\ln (B(s)|t| / 2)+\ln \left(1+8 /\left(B(s) \Lambda^{2}\right)\right)\right. \\
& \left.+\left(4|t| / \Lambda^{2}\right) \ln \left(4|t| / \Lambda^{2}\right)+2|t| / \Lambda^{2}\right],
\end{aligned}
$$

where $\Lambda=0.71 \mathrm{GeV}^{2}$. For the dypol form of the form factor it is need take some approximation with $\Lambda=0.71 / 4 \mathrm{GeV}^{2}$.

The calculations of the phase factor beyond the limit $t \rightarrow 0$ was carried out in 27.

In the absence of nuclear forces, we have only the Coulomb amplitude. In the second Born approximation there appear the additional phase, $\varphi(t)_{C}$ which are sufficiently complicated but eventually exactly calculable (see refs. [27, 28, 29] for details). As a 
Table 1 Proton-antiproton scattering (the phase dependence)

\begin{tabular}{|c|c|c|c|c|c|c|}
\hline$p_{L}\left(\frac{G e V}{c}\right)$ & $\mathrm{N}$ & $\rho_{\text {exp. }}$ & $\rho_{\varphi(\text { Born }), n=1}$ & $\rho_{\varphi(\text { our }), n=1}$ & $\rho_{\varphi(\text { our }), n-\text { free }}$ & $\mathrm{n}$ \\
\hline 3.702 & 134 & +0.018 & $+0.046 \pm 0.005$ & $+0.033 \pm 0.01$ & $-0.004 \pm 0.005$ & 1.08 \\
\hline 4.066 & 34 & -0.015 & $+0.038 \pm 0.008$ & $+0.026 \pm 0.01$ & $-0.039 \pm 0.02$ & 1.15 \\
\hline 5.603 & 215 & -0.047 & $+0.004 \pm 0.003$ & $-0.004 \pm 0.003$ & $-0.037 \pm 0.015$ & 1.06 \\
\hline 5.724 & 115 & -0.051 & $+0.014 \pm 0.01$ & $+0.002 \pm 0.004$ & $-0.053 \pm 0.012$ & 1.12 \\
\hline 5.941 & 140 & -0.063 & $-0.002 \pm 0.005$ & $-0.012 \pm 0.004$ & $-0.075 \pm 0.013$ & 1.13 \\
\hline 6.234 & 34 & -0.06 & $-0.016 \pm 0.004$ & $-0.028 \pm 0.02$ & $-0.007 \pm 0.02$ & 0.96 \\
\hline
\end{tabular}

result, for the total Coulomb scattering amplitude, we have the eikonal approximation of the second order in $\alpha\left(=e^{2} / h c\right)$

$$
F_{C}(q) \quad=F_{C}^{1 B}+F_{C}^{2 B}=-\frac{\alpha}{q^{2}}\left[\frac{\Lambda^{4}}{\left(\Lambda^{2}+q^{2}\right)^{2}}\right]\left[1+i \alpha\left(\left\{\ln \left(\frac{\lambda^{2}}{q^{2}}\right)+\nu_{s}\right\}\right],\right.
$$

where

$$
\nu_{s}=A \ln \left(\frac{\left(\Lambda^{2}+q^{2}\right)^{2}}{\Lambda^{2} q^{2}}\right)+B \ln \left(\frac{4 \Lambda^{2}}{\left(\sqrt{\left(4 \Lambda^{2}+q^{2}\right.}+q\right)^{2}}\right)+C .
$$

The coefficients $A, B, C$ are defined in [29].

$$
\begin{aligned}
& A=\frac{q^{2}\left(2 \Lambda^{2}+q^{2}\right)}{\Lambda^{4}} ; \quad C=\frac{2 \Lambda^{4}-17 \Lambda^{2} q^{2}-q^{4}}{\left(4 \Lambda^{2}+q^{2}\right)^{2}} ; \\
& B=\frac{\left(\Lambda^{2}+q^{2}\right)^{2}\left[4 \Lambda^{4}\left(\Lambda^{2}+7 q^{2}\right)+q^{4}\left(10 \Lambda^{2}+q^{2}\right)\right]}{\Lambda^{4} q\left(4 \Lambda^{2}+q^{2}\right)^{5 / 2}} .
\end{aligned}
$$

The impact of the spin of the scattered particles was analyzed in [19, 30] by using the eikonal approach for the scattering amplitude. Using the helicity formalism for high energy hadron scattering in [19] it was shown that at small angles, all the helicity amplitudes have the same $\varphi(s, t)$.

\section{Impact of the Coulomb-hadron interference phase}

Let us define the hadronic spin-non-flip amplitudes as

$$
F_{\mathrm{nf}}^{h}(s, t)=\left[F_{1}^{h}(s, t)+F_{3}^{h}(s, t)\right] / 2
$$

Equations (15) were applied at high energies and at small momentum transfer, with the following usual assumptions for helicity amplitudes: $\phi_{1}=\phi_{3}, \phi_{2}=\phi_{4}=0$; and, as usual, we neglect in this section the possible contribution of the spin-flip amplitude.

We now compare fits to the experimental $p \bar{p}$-scattering [18, 31, 32, 33] data at low energies with different approximations for the Coulomb-hadron interference phase factor (see also [34]). Here we also include the additional normalization of the differential cross sections $n$ which reflects the systematical errors of the luminosity. First, we use the simple form of the phase, eq. (10), with taking into account the $n=1$. The values obtained for $\rho(s, t=0)$ are shown in the second column of Table 1. The results lie above the values of $\rho(s)$ extracted during the experiments. 
If we take a slightly more complicated phase, eq.(11), with the dipole form factor and our correction term, eq, 12 , taking into account the two photon approximation and the dipole form factor, the new fitting procedure with $n=1$ gives the different sizes of $\rho(s)$ (3 column of Table 1). Now the results slightly decrease but still lie above

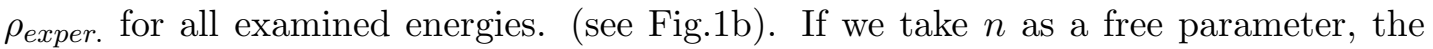
obtained size of $\rho$ more decrease as well, in two cases $\left(p_{L}=3.7\right.$ and $\left.4.066 \mathrm{GeV} / \mathrm{c}\right)$ lie slightly lower then the $\rho_{\text {exper. }}$. However, in this case we obtain large corrections of the normalization of the experimental data (up to 15\%). It is shown that the form of the differential cross sections is far away from the simple exponential dependence.

\section{The slope of the hadron spin-flip amplitude}

As it is not possible to calculate exactly the hadronic amplitudes from first principles, we have to resort to some assumptions for what concerns their form ( $s$ and $t$ dependence) [35, 36, 37]. Let us define the slope of the scattering amplitude as the derivative of the logarithm of the amplitudes with respect to $t$. For an exponential form of the amplitudes this coincides with the usual slope of the differential cross sections divided by 2 .

In most analyses, one makes the assumptions that the imaginary and real parts of the spin-non-flip amplitude have an exponential behavior with the same slope and that the imaginary and real parts of the spin-flip amplitudes, without the kinematic factor $\sqrt{|t|}[38$, are proportional to the corresponding parts of the non-flip amplitude.

That is not so as regards the $t$ dependence was shown in Ref. [39], where $F_{s f}^{h}$ is multiplied by a phenomenological $t$-dependent function. Moreover, one usually takes the ratio of the spin-flip parts to the spin-non-flip parts of the scattering amplitude to be energy independent. All this is our theoretical uncertainty [37, 40]. In [41] it was shown that in the case $B^{-}=2 B^{+}$the contribution of the spin-flip amplitude can be felt in the differential cross sections of the elastic hadron scattering at small momentum transfer.

According to the standard interpretation, the hadron spin-flip amplitude is connected with quark exchange between the scattering hadrons, and at large energy and small angles it can be neglected. Some models, which take into account nonperturbative effects, lead to the non-dying hadron spin-flip amplitude [42, 43, 44, 45, 46]. Another complicated question is related to the difference in phases of the spinnon-flip and spin-flip amplitude.

We introduce the small- $t$ spin non-flip amplitude in the form

$$
\operatorname{Im} F_{n f}^{h}(s, t) \sim \exp \left(B_{1}^{+} t\right), \quad \operatorname{Re} F_{n f}^{h}(s, t) \sim \exp \left(B_{2}^{+} t\right),
$$

and we define the "residual" spin flip amplitude where the kinematical vanishing at $t=0$ has been removed.

$$
\operatorname{Im}_{\tilde{F_{s f}^{h}}}(s, t)=\frac{1}{\sqrt{|t|}} \operatorname{Im} \phi_{5}^{h}(s, t) \sim \exp \left(B_{1}^{-} t\right) ; \quad \operatorname{Re}_{F_{s f}^{h}}(s, t)=\sim \exp \left(B_{2}^{-} t\right) .
$$

At small $t\left(\sim 0 \div 0.1 \mathrm{GeV}^{2}\right)$, practically all semiphenomenological analyses assume:

$$
B_{1}^{+} \approx B_{2}^{+} \approx B_{1}^{-} \approx B_{2}^{-} .
$$

Actually, if we take the eikonal representation for the helicity amplitudes

$$
F_{n f}^{h}(s, t)=-i p \int_{0}^{\infty} b d b J_{0}(q b)\left[e^{\chi_{0}(s, b)}-1\right]
$$




$$
F_{s f}^{h}(s, t)=-i p \int_{0}^{\infty} b d b J_{1}(q b) \chi_{1}(s, b) e^{\chi_{0}(s, b)} .
$$

where

$$
\begin{aligned}
& \chi_{0}(s, b)=\frac{1}{2 i p} \int_{-\infty}^{\infty} d z V_{0}(\vec{b}, z) \\
& \chi_{1}(s, b)=\frac{b}{2 i p} \int_{-\infty}^{\infty} d z V_{1}(\vec{b}, z)
\end{aligned}
$$

If the potentials $V_{0}$ and $V_{1}$ are assumed to have a Gaussian form

$$
V_{0,1}(b, z) \sim \int_{-\infty}^{\infty} \frac{1}{4 B \sqrt{\pi B}} e^{r^{2} /(4 B)} d z=\frac{1}{2 B} e^{-b^{2} /(4 B)}
$$

where $r^{2}=b^{2}+z^{2}$. In the first Born approximation $\phi_{1}^{h}$ and $\hat{\phi}^{h}{ }_{5}$ will have the same slope

$$
\begin{gathered}
F_{n f}^{h}(s, t) \sim \int_{0}^{\infty} b d b J_{0}(q b) e^{-b^{2} /(4 B)} /(2 B)=e^{-B q^{2}} \\
F_{s f}^{h}(s, t) \sim \int_{0}^{\infty} b^{2} d b J_{1}(q b) e^{-b^{2} /(4 B)} /(2 B)=2 q B e^{-B q^{2}} .
\end{gathered}
$$

In this special case, therefore, the slopes of the spin-flip and "residual"spin-non-flip amplitudes are indeed the same.

The first observation that the slopes don't coincide was made in [48, 49]. It was found from the analysis of the $\pi^{ \pm} p \rightarrow \pi^{ \pm} p$ and $p p \rightarrow p p$ reactions at $p_{L}=20 \div$ $30 \mathrm{GeV} / \mathrm{c}$ that the slope of the "residual" spin-flip amplitude is about twice as large as the slope of the spin-non flip amplitude. This conclusion was confirmed by the phenomenological analysis carried out in [8] for spin correlation parameters of the elastic proton-proton scattering at $p_{L}=6 \mathrm{GeV} / \mathrm{c}$.

The model-dependent analysis based on all the existing experimental data of the spin-correlation parameters above $p_{L} \geq 6 \mathrm{GeV}$ allows us to determine the structure of the hadron spin-flip amplitude at high energies and to predict its behavior at superhigh energies [47]. This analysis shows that the ratios $\operatorname{Re} \phi_{5}^{h}(s, t) /\left(\sqrt{|t|} \operatorname{Re} \phi_{1}^{h}(s, t)\right)$ and $\operatorname{Im} \phi_{5}^{h}(s, t) /\left(\sqrt{|t|} \operatorname{Im} \phi_{1}^{h}(s, t)\right)$ depend on $s$ and $t$. At small momentum transfers, it was found that the slope of the "residual" spin-flip amplitudes is approximately twice the slope of the spin-non flip amplitude.

Let us see what we obtain in the case of an exponential tail for the potentials [36]. If we take

$$
\chi_{i}(s, b) \sim H e^{-a b},
$$

and use the standard integral representation

$$
\int_{0}^{\infty} x^{\alpha-1} \exp (-p x) J_{\nu}(c x) d x=I_{\nu}^{\alpha}
$$

with

$$
I_{\nu}^{\nu+2}=2 p(2 c)^{\nu} \Gamma(\nu+3 / 2) 1 /\left[\sqrt{\pi}\left(p^{2}+c^{2}\right)^{3 / 2}\right]
$$

we obtain

$$
F_{n f}(s, t)=\int \rho d \rho e^{-a \rho} J_{0}(\rho q)=\frac{a}{\left(a^{2}+q^{2}\right)^{3 / 2}} \approx \frac{1}{a \sqrt{a^{2}+q^{2}}} e^{-B q^{2}}
$$


with $B=1 / a^{2}$, where we have used the approximation

$$
1 /(1+x) \sim(1-x) \sim \exp (-x) .
$$

For the "residual" spin-flip amplitude, on the other hand, we obtain

$$
\sqrt{|t|} \tilde{F}_{s f}(s, t)=\int b^{2} d b e^{-a b} J_{1}(q b)=\frac{3 a q}{\left(a^{2}+q^{2}\right)^{5 / 2}} \approx \frac{3 a q B^{2}}{\sqrt{a^{2}+q^{2}}} e^{-2 B q^{2}}
$$

In this case, therefore, the slope of the "residual" spin-flip amplitude exceeds the slope of the spin-non-flip amplitudes by a factor of two.

Hence, a long tail hadron potential implies a significant difference of the slopes of the "residual" spin-flip and of the spin-non-flip amplitudes. Note, that the procedure of eikonalization would lead to a further increase of the difference of these two slopes.

\section{Impact of the spin-flip contribution}

In [50], it is claimed that the analysis of the low energy experimental data is not affected by the spin-flip amplitude on the extracted value of $\rho(s, t)$. We believe that this result must be checked up carefully and that at low energies the size of the spin-flip amplitude is non negligible. In this work, we examine a simple model for the spin-flip amplitude and try to find its impact on the determination of $\rho(s, t)$ from the low energy data of proton-proton scattering. Based on the analysis carried out in the previous section we take the spin non-flip and spin flip amplitudes in the simplest exponential form

$$
F_{n f}^{h}=h_{n f}[i+\rho(s, t=0)] e^{B^{+} t / 2}
$$

and

$$
F_{s f}^{h}=\sqrt{-t} / m_{p} h_{s f}[i+\rho(s, t=0)] e^{B^{-} t / 2},
$$

with $B^{-}=2 B^{+}$. and the full amplitudes will include the corresponding electromagnetic parts and the Coulomb-hadron phase factors as analyzed previously.

The results of our new fits of the proton-antiproton experimental data at $p_{L}=$ 3.7..6.2 GeV/c are presented in Table 2. The changes of $\sum_{i} \chi_{i}^{2}$ after including the contribution of the spin-flip amplitude are reflected by the coefficient

$$
R_{\sum_{i} \chi_{i}^{2}}=\frac{\sum_{i} \chi_{i \text { without sf. }}^{2}-K \sum_{i} \chi_{i \text { with sf. }}^{2} .}{\sum_{i} \chi_{i}^{2} \text { without sf. }} .
$$

where $K=\left(N-m_{1}\right) /\left(N-m_{2}\right)$ is the coefficient taking into account the difference of the number of free parameters $\left(m_{1}=3\right.$ for the case without spin-flip contribution and $m_{2}=4$ with taking into account the spin-flip contribution).

We obtain the sizes of $\rho(s, t=0)$ close to zero and prevalently positive. The contribution of the spin-flip amplitude is sizeable and impacts the extracted values of $\rho(s, t=0)$. Most remarkable is that the obtained size of the constant of the spin-flip amplitude coincides for practically all examined energies. These constants are pretty large and have sufficiently small errors. This shows that a careful analysis of the size of $\rho$ requires to take into account the contribution of the spin-flip amplitude to the differential cross section at small angles, at least at not too high energies.

The corresponding analysis was made for the proton-proton elastic scattering (see Table 3). Again the size of the hadron spin-flip amplitude is determined sufficiently well. The changes of the size of $\rho(s, t=0)$ are $5 \% \div 10 \%$. However, as these experimental data have larger errors then in the previous data of the proton-antiproton scattering, the determination of the spin-flip amplitude is worst and the impact its contribution on the determined size of the $\rho(s, t=0)$ is small. 
Table 2 Proton-antiproton elastic scattering (the spin dependence)

\begin{tabular}{|c|c|c|c|c|c|} 
& \multicolumn{5}{|c|}{} \\
\hline$p_{L(\mathrm{GeV} / \mathrm{c})}$ & $\mathrm{N}$ & $\rho_{\text {exp. }}$ & $R_{\chi^{2}}$ & $\rho_{\text {model }}$ & $h_{s f}, \mathrm{GeV}$ \\
\hline 3.702 & 134 & $+0.018 \pm 0.014$ & $7.3 \%$ & $+0.055 \pm 0.001$ & $38.3 \pm 0.4$ \\
4.066 & 34 & $-0.015 \pm 0.024$ & $22.5 \%$ & $+0.061 \pm 0.02$ & $45.4 \pm 6.7$ \\
5.603 & 215 & $-0.047 \pm 0.017$ & $3.1 \%$ & $+0.002 \pm 0.005$ & $31.1 \pm 3.7$ \\
5.724 & 115 & $-0.051 \pm 0.011$ & $5.7 \%$ & $+0.023 \pm 0.001$ & $30.8 \pm 0.4$ \\
5.941 & 140 & $-0.063 \pm 0.015$ & $3.8 \%$ & $+0.009 \pm 0.016$ & $38.6 \pm 0.4$ \\
\hline
\end{tabular}

Table 3 Proton-proton elastic scattering (the spin dependence)

\begin{tabular}{|c|c|c|c|c|c|}
\hline$p_{L(G e V / c)}$ & $\mathrm{N}$ & $\rho_{\text {exp. }}$ & $R_{\chi^{2}}$ & $\rho_{\text {model }}$ & $h_{s f}, \mathrm{GeV}$ \\
\hline 9.4 & 34 & $-0.372 \pm 0$ & $1.2 \%$ & $-0.413 \pm 0.02$ & $32.1 \pm 15.2$ \\
\hline 18.9 & 67 & $-0.266 \pm 0.008$ & $4.8 \%$ & $-0.283 \pm 0.011$ & $7.8 \pm 1.7$ \\
\hline 38. & 65 & $-0.161 \pm 0.006$ & $2.6 \%$ & $-0.169 \pm 0.007$ & $6.9 \pm 1.7$ \\
\hline 39.4 & 47 & $-0.195 \pm 0.015$ & $0.02 \%$ & $-0.201 \pm 0.01$ & $15.76 \pm 5$ \\
\hline 40.6 & 65 & $-0.159 \pm 0.007$ & $0.1 \%$ & $-0.17 \pm 0.01$ & $12.9 \pm 1.2$ \\
\hline 69.5 & 73 & $-0.1066 \pm 0.011$ & $3.6 \%$ & $-0.119 \pm 0.015$ & $8.5 \pm 0.2$ \\
\hline
\end{tabular}

\section{Additional effects (oscillation)}

Previously we have examined various standard corrections to the exponential behavior of the elastic scattering amplitude. However, some additional corrections connected with non-standard contributions to the main exponential amplitude should be taken into account, such as bumps in the momentum transfer [51], or small-t additional oscillations in the scattering amplitude [52, 53. Such oscillations were noted at high energy in the analysis of the UA4/2 Collaboration [54. Recently many other experimental data have been analyzed for the presence of oscillations [5, 55]. The analysis points to the possible existence of oscillations in the low energy experimental data of the proton-antiproton scattering at $3.8 \leq p_{L} \leq 6.2 \mathrm{GeV}[55]$.

Let us try to simulate additional oscillations of the elastic scattering amplitude in the form

$$
F_{o s c}^{h}(t)=h_{o s c} \sin \left[\pi\left(\phi_{0}+q\right) / q_{0}\right] G_{d}^{2}(t) .
$$

where $G_{d}(t)$ is the standard dipole form factor, $\phi_{0}$ is the starting phase, and $q_{0}$ is momentum transfer determining the period of the oscillation function (order $\sim 0.015 \mathrm{GeV}$ ). The largest contribution comes from the interference of this amplitude with the electromagnetic part of the scattering amplitude. The inclusion of such an additional amplitude essentially decreases $\chi^{2}$ in the analysis of the proton-antiproton elastic scattering (see $R_{\chi^{2}}$ in Table 4 , where $R_{\chi^{2}}$ is defined in eq.(26). The decreasing that occurs for proton-proton scattering (see, Table 5) less pronounced, but still visible. Practically in all cases the sizes of the spin-flip amplitude and the additional oscillation part are determined well. The new values of $\rho(s, t=0)$ for the proton-proton scattering lie slightly lower than the experimental data. For the proton-antiproton the new sizes of $\rho(s, t=0)$ are slightly above the experimental data (Fig.2). 
Table 4 Proton-antiproton elastic scattering (the spin dependence + oscillations)

\begin{tabular}{|c|c|c|c|c|c|c|} 
& \multicolumn{7}{|c|}{$\rho_{\text {exp. }}$} & $R_{\chi^{2}}$ & $\rho_{\text {model }}$ & $h_{s f}, \mathrm{GeV}$ & $h_{\text {osc. }} 10^{2}, \mathrm{GeV}$ \\
\hline 3.702 & 134 & +0.018 & $12.7 \%$ & $+0.044 \pm 0.01$ & $34.7 \pm 6.5$ & $9.1 \pm 3.7$ \\
4.066 & 34 & -0.015 & $28.1 \%$ & $+0.055 \pm 0.02$ & $44.6 \pm 6.5$ & $9.1 \pm 3.7$ \\
5.603 & 215 & -0.047 & $19.5 \%$ & $-0.007 \pm 0.005$ & $12.7 \pm 0.6$ & $11.2 \pm 2.3$ \\
5.724 & 115 & -0.051 & $20.1 \%$ & $+0.007 \pm 0.005$ & $23.1 \pm 0.9$ & $13.9 \pm 1.4$ \\
5.941 & 140 & -0.063 & $20.4 \%$ & $-0.014 \pm 0.002$ & $24.7 \pm 0.6$ & $14.5 \pm 0.4$ \\
\hline
\end{tabular}

Table 5 Proton-proton elastic scattering (the spin dependence + oscillations)

\begin{tabular}{|c|c|c|c|c|c|c|} 
& \multicolumn{7}{|c|}{${ }^{2}\left(\frac{\mathrm{GeV}}{c}\right)$} & $\mathrm{N}$ & $\rho_{\text {exp. }}$ & $R_{\chi^{2}}$ & $\rho_{\text {model }}$ & $h_{s f}, \mathrm{GeV}$ & $h_{\text {osc. }} 10^{2}, \mathrm{GeV}$ \\
& & & & & & \\
\hline 9.4 & 34 & -0.372 & $1.2 \%$ & $-0.41 \pm 0.028$ & $35.8 \pm 13.7$ & $10.8 \pm 4.7$ \\
18.9 & 67 & -0.266 & $5.8 \%$ & $-0.28 \pm 0.0117$ & $7.8 \pm 1.8$ & $7.1 \pm 2.9$ \\
38. & 65 & -0.161 & $2.3 \%$ & $-0.166 \pm 0.007$ & $6.9 \pm 1.7$ & $738 \pm 0.5$ \\
39.4 & 47 & -0.195 & $8.7 \%$ & $-0.19 \pm 0.016$ & $12.1 \pm 3.8$ & $7.38 \pm 2.9$ \\
40.6 & 65 & -0.159 & $8.2 \%$ & $-0.157 \pm 0.011$ & $11.5 \pm 4.1$ & $5.9 \pm 2.1$ \\
69.5 & 73 & -0.1066 & $19.3 \%$ & $-0.176 \pm 0.002$ & $8.2 \pm 0.1$ & $31.4 \pm 2.4$ \\
\hline
\end{tabular}

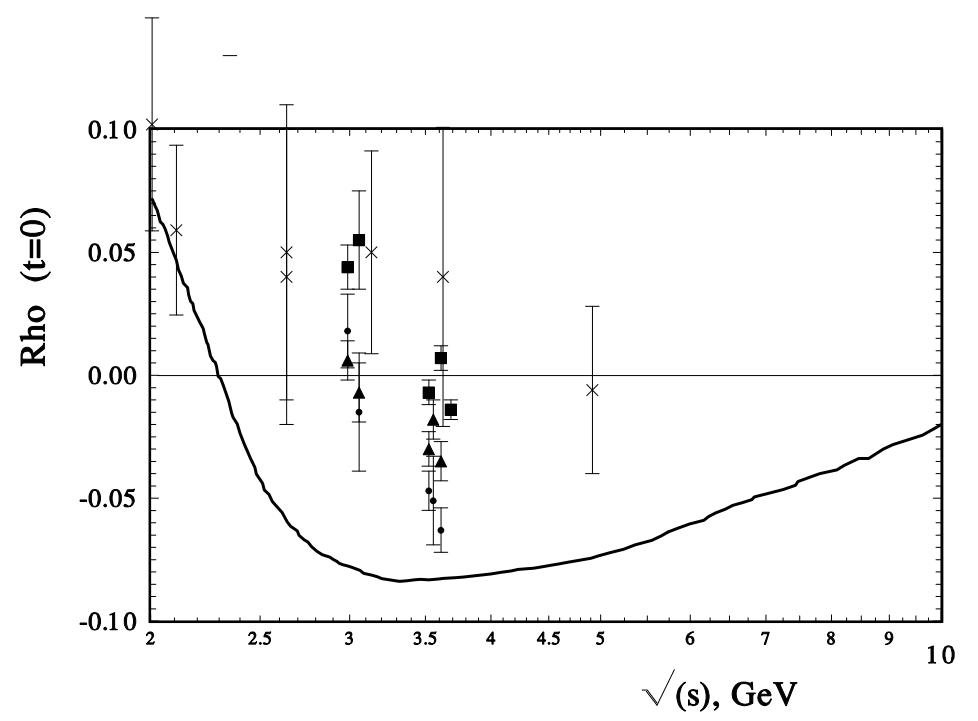

Figure 2: $\rho(s, t=0)$ of the $p \bar{p}$ scattering at low energies (circles and points, as in Fig.1(b), squares - our extraction, see Table 4. 


\section{Conclusion}

Future new data from the LHC experiments will hopefully give the possibility to carry out new analysis of the dispersion relation leading to new effects such as, for example, a fundamental length of an order of TeV. However, for such analysis one also needs the knowledge of the low energy data with high accuracy. As we have seen, the existing forward-scattering data at $p_{L}=4 \div 60(\mathrm{GeV})$ of the size $\rho(s, t)$ contradict each other and the $\rho(s, t)$ - data of the proton-antiproton scattering at $p_{L}=3.7 \div 6.2(\mathrm{GeV})$ contradict the dispersion relations prediction in form [1]. The present analysis, which includes the contributions of the different correction terms in most part support the data of $\rho(s, t=0)$ obtained in [18] and show a larger difference with predictions of [1] and coincide with some phenomenological analysis based on the dispersion relations carried out in [56, 57]. However, the last works include in his phenomenological analysis with many free parameters for low energy region the data of the proton-antiproton scattering at $p_{L}=3.7 \div 6.2(\mathrm{GeV})$.

It is needed to note that taking into account the contribution of the spin-flip amplitude improves the description of the differential cross sections and decreases $\chi^{2}$. Likely, however, an additional analysis is needed to include additional corrections connected with the possible oscillation in the scattering amplitude and with the $t$-dependence of the spin-flip scattering amplitude. We hope that the forward experiments at NICA can give valuable information for the improvement of our theoretical understanding of the strong hadrons interaction. This is especially true for the experiment at NICA with polarized beams.

Acknowledgement: The author would like to thank J. -R. Cudell and E. Predazzi for helpful discussions, gratefully acknowledges the financial support from BELSPO and would like to thank the University of Liège where part of this work was done.

\section{References}

[1] P. Kroll, W. Schweiger, Nucl.Phys. A503 (1089) 865.

[2] V. Barone, E. Predazzi, " High Energy Particle Diffraction", NY (2002).

[3] A.Martin, F. Cheung, Analytic properties and bounds of the scattering amplitude, (Cordon and Breach, New York, 1970).

[4] S.M.Roy, Phys.Rep. C 5 (1972) 125 .

[5] R. Fiore, L. Jenkovszky, R. Orava, E. Predazzi, A. Prokudin, O. Selyugin, Mod.Phys., A24: (2009) 2551.

[6] N.N. Khuri, Proceedings of Les Rencontre de Physique de la Villee d'Aoste: Results and perspectives in Particle Physics (M. Greeco ed.), p.701 Gif-nur-Yvette, France (1994).

[7] C. Bourrely, N.N. Khuri, A. Martin, J. Soffer, T.T. Wu, Proceedings EDS 2005, Blois, France (2005).

[8] M.Sawamoto, S.Wakaizumi, Proc Theor.Phis. 62 (1979) p.1293.

[9] O.V. Selyugin, J.-R. Cudell, E. Predazzi, Eur.Phys.J.ST 162, (2008) 37-42.

[10] O. Selyugin, Phys. Lett. B333, 245 (1994) 
[11] O. Selyugin, Sov. J. Nucl. Phys. 55, 466 (1992)

[12] J. R. Cudell et al. [COMPETE Collaboration], Phys. Rev. D 65 (2002) 074024.

[13] J. R. Cudell et al. [COMPETE Collaboration], Phys. Rev. Lett. 89 (2002) 201801.

[14] J. -R. Cudell, V. Ezhela, K. Kang, S. Lugovsky and N. Tkachenko, Phys. Rev. D 61 (2000) 034019 [Erratum-ibid. D 63 (2001) 059901] [arXiv:hep-ph/9908218].

[15] D. Gross, et al., Phys.Rev.Lett. 41, (1978) 217.

[16] A.A. Kuznetzov et al., preprint JINR P1-80-376, Dubna (1980).

[17] L.A. Fajardo et al., Phys.Rev., D24 (1981) 46.

[18] T.A. Armstrong et al., Phys.Lett. B 385 (1996) 479.

[19] N. H. Buttimore, E. Gotsman, E. Leader, Phys. Rev. D 35, (1987) 407.

[20] A.I. Akhiezer, I.Ya. Pomeranchuk, J. Phys. 9 (1945) 471.

[21] H. Bethe, Ann. Phys. 3, (1958) 190;

[22] L.D. Soloviev, Zh.Eksp.Teor.Fiz., 49,(1965) 292.

[23] J. Rix, R.M. Thaler, Phys. Rev., 152, (1966)1357.

[24] M.P. Locher, Nucl.Phys., B 2, (1967) 525.

[25] G. B. West, D. R. Yennie, Phis. Rev. 172, (1968) 1414.

[26] R. Cahn, Zeitschr. fur Phys. C 15, (1982) 253.

[27] O.V. Selyugin, Mod. Phys. Lett. A11 (1996) 2317.

[28] O.V. Selyugin, Mod. Phys. Lett. A12, (1997) 1379.

[29] O. V. Selyugin, Phys. Rev. D 60 (1999) 074028

[30] L.I. Lapidus, Particles \& Nuclei 9, (1978) 84.

[31] S. Trokenheim, Fermilab-Thesis-1995-40,(1995).

[32] Durham HepData Project, M.R. Whalley, http://durpdg.dur.ac.uk/hepdata/reac.html.

[33] K.R. Schubert, In Landolt-Börnstein, New Series, v. 1/9a, (1979).

[34] J.-R. Cudell, E. Predazzi, and O.V. Selyugin, Proceedings of the Int.Conf. "High energy spin physics", ed. A.V. Efremov, Dubna, (2011).

[35] O.V. Selyugin, Proceedings of the Int.Conf. "New Trends in High Energy Physics", ed. P.N.Bogolyubov, L.Jenkovszkky,Yalta(Crimea), September 22-29, 2001, Kiev (2001), p. 237.

[36] E. Predazzi, O.V. Selyugin, Eur.Phys.J. A 13 , (2002) 471-475.

[37] J.-R. Cudell, E. Predazzi, and O.V. Selyugin, Eur. Phys. J. A 21 (2004) 479-486.

[38] N.H. Buttimore et al., Phys.Rev. D 59 (1999) 114010.

[39] C. Bourrely, J. Soffer, hep-ph/9611234.

[40] A.F. Martini, and E. Predazzi, Phys. Rev. D 66 (2002) 034029.

[41] O.V. Selyugin, Mod.Phys.Lett, A 14, (1999) 223.

[42] B.Z. Kopeliovich and B.G. Zakharov, Phys.lett. B226 (1989) 156.

[43] M. Anselmino and S. Forte, Phys. Rev. Lett. 71, (1993) 223.

[44] A.E. Dorokhov, N.I. Kochelev and Yu.A. Zubov, Int. Jour. Mod. Phys. A8, (1993) 603. 
[45] N. Akchurin, S.V. Goloskokov, O.V. Selyugin, Int. J. Mod. Phys. A 14 (1999) 252.

[46] J.R. Cudell, E. Predazzi, O.V. Selyugin, Particles\&Nuclei, 36(7) (2004) 132.

[47] O.V.Selyugin, Phys. of Atomic Nuclei 62 (1999) 333.

[48] E. Predazzi, G. Soliani, Nuovo Cim. A 2 (1967) 427.

[49] K. Hinotani, H.A. Neal, E. Predazzi and G. Walters, Nuovo Cim., A 52 (1979) 363.

[50] S.B. Nurushev and V.A. Okorokov, Proceedings XII Workshop on high energy spin physics, DSPIN-2007, Sept. 3-7, Dubna (2007), Dubna, 2008, p. 117.

[51] S. Barshay, P. Heiliger, Z. Phys. C 64675 (1994).

[52] N. I. Starkov, V. A. Tsarev, Pisma Zh. Eksp. Teor. Fiz. 23, (1976) 403.

[53] O.V. Selyugin, in Hadrons-95, Proc. of the XIth Workshop on Soft Physics, edited by L. Jenkovszky, Kiev, (1995), p.65.

[54] P. Gauron, B. Nicolescu, O.V. Selyugin, Phys.Lett. 397305 (1997).

[55] O.V. Selyugin, J.-R. Cudell, in the International Workshop on Diffraction in HighEnergy Physics, Otranto (Lecce, Italy), September 10-15, 2010; AIP Conf. Proc. 1350:115-118, 2011; ArXiv:hep-ph/1011.4177.

[56] J.R. Cudell, E. Martynov, O.Selyugin, A. Lengyel, Phys.Lett. B 58778 (2004).

[57] J.R. Cudell, E. Martynov, O.Selyugin, EPJ C33 s 533 (2004). 